\title{
Dicer-Like 4 Is Involved in Restricting the Systemic Movement of Zucchini yellow mosaic virus in Nicotiana benthamiana
}

\author{
Teresa Cordero, ${ }^{1}$ Lidia Cerdán, ${ }^{1}$ Alberto Carbonell, ${ }^{1}$ Konstantina Katsarou, ${ }^{2}$ Kriton Kalantidis, ${ }^{2}$ and \\ José-Antonio Daròs ${ }^{1}$ \\ ${ }^{1}$ Instituto de Biología Molecular y Celular de Plantas (Consejo Superior de Investigaciones Científicas-Universidad Politécnica \\ de Valencia), 46022 Valencia, Spain; and ${ }^{2}$ Institute of Molecular Biology and Biotechnology, Foundation for Research and \\ Technology; and Department of Biology, University of Crete, Heraklion, Crete, Greece
}

Accepted 12 December 2016.

\begin{abstract}
Zucchini yellow mosaic virus (ZYMV) induces serious diseases in cucurbits. To create a tool to screen for resistance genes, we cloned a wild ZYMV isolate and inserted the visual marker Roseal to obtain recombinant clone ZYMV-Ros1. While in some plant-virus combinations Rosea1 induces accumulation of anthocyanins in infected tissues, ZYMV-Ros1 infection of cucurbits did not lead to detectable anthocyanin accumulation. However, the recombinant virus did induce dark red pigmentation in infected tissues of the model plant Nicotiana benthamiana. In this species, ZYMV-Ros1 multiplied efficiently in local inoculated tissue but only a few progeny particles established infection foci in upper leaves. We used this system to analyze the roles of Dicer-like (DCL) genes, core components of plant antiviral RNA silencing pathways, in ZYMV infection. ZYMV-Ros1 local replication was not significantly affected in single $D C L$ knockdown lines nor in double $D C L 2 / 4$ and triple DCL2/3/4 knockdown lines. ZYMV-Ros1 systemic accumulation was not affected in knockdown lines $D C L 1, D C L 2$, and DCL3. However in DCL4 and also in DCL2/4 and DCL2/3/4 knockdown lines, ZYMV-Ros1 systemic accumulation dramatically increased, which highlights the key role of $\mathrm{DCL} 4$ in restricting virus systemic movement. The effect of $D C L 4$ on ZYMV systemic movement was confirmed with a wild-type version of the virus.
\end{abstract}

When plant viruses manage to enter an initial cell in their hosts, frequently with the help of a vector organism, they express and replicate their genomes to produce progeny that first move cell to cell to reach the host vascular tissue and then move long distance to distal areas. During this process, viruses hijack multiple elements, complexes, and structures from host plants. They must also surpass all barriers and neutralize plant defensive responses. Viral and host components establish a complex interaction network that frequently leads to infection and disease

Nucleotide sequence data for the Vera isolate of Zucchini yellow mosaic virus has been deposited in the GenBank database under accession number KX499498.

Corresponding author: J. A. Daròs; E-mail: jadaros@ibmcp.upv.es

*The $\boldsymbol{e}$-Xtra logo stands for "electronic extra" and indicates that six supplementary figures and three supplementary tables are published online.

○ 2017 The American Phytopathological Society response but to resistance at other times. One of the main goals of plant virology is to understand this network in order to be able to modify the equilibrium to favor resistance.

Plants use RNA-mediated gene silencing pathways to fight invading viruses (Hamilton and Baulcombe 1999). Plant Dicerlike (DCL) RNases recognize virus-specific double-stranded or highly-structured RNAs to produce 21- to 24-nucleotide (nt) small interfering RNA (siRNA) duplexes (Aliyari and Ding 2009; Zhang et al. 2015). One of the strands is selectively loaded by an argonaute protein to guide the RNA-induced silencing complex to target and repress sequence complementary viral RNAs (Carbonell and Carrington 2015). The amount of viral double-stranded RNA that triggers the antiviral RNA silencing pathways in the host plant is amplified by RNAdependent RNA (RDR) polymerase activities by using viral siRNAs as primers (Wang et al. 2010). In order to counteract this defensive response, evolution has shaped the proteins dedicated to suppress RNA silencing in virtually all plant viruses (Csorba et al. 2015).

Zucchini yellow mosaic virus (ZYMV) is a prominent pathogen of many plant species of the family Cucurbitaceae, which includes different important crops (Lecoq and Desbiez 2012). It was first isolated in Italy in 1973 (Lisa et al. 1981), although it is currently present worldwide (Desbiez and Lecoq 1997). ZYMV belongs to the genus Potyvirus in the family Potyviridae and its genome consists of approximately 10,000-nt-long single-stranded RNA molecules of plus polarity that encodes two versions of a large polyprotein (Wu et al. 2010). The genomic RNA of potyviruses (genus Potyvirus) is covalently attached, at its 5' end, to a viral protein genome-linked (VPg), contains a polyadenylated tail at its $3^{\prime}$ end, and is encapsidated by approximately 2,000 units of the viral coat protein (CP) in an elongated and flexuous virion (Revers and García 2015). Potyviral proteins, which include the P1 protease, the helper component protease (HC-Pro), the P3 protein and P3N-PIPO, small hydrophobic polypeptide $6 \mathrm{~K} 1$, the cylindrical inclusion (CI) protein, a second small hydrophobic polypeptide $6 \mathrm{~K} 2$, the nuclear inclusion $a$ (NIa) protein, which is further split into its two VPg and protease (NIaPro) domains, viral RDR polymerase or nuclear inclusion $b(\mathrm{NIb})$ protein, and the CP (Fig. 1A), are produced from a regulated cascade of proteolytic processing through the activity of three viral proteases, namely, P1, HC-Pro, and NIaPro.

For the purpose of obtaining a viral clone to facilitate screening for resistance in cucurbitaceous plants, we cloned a wild isolate of ZYMV and constructed a recombinant clone that 
expresses the snapdragon (Antirrhinum majus L.) Roseal marker gene, which activates anthocyanin (a class of flavonoid pigments) biosynthesis and allows the visual tracking of viral infection in different plant species (Bedoya et al. 2012). Anthocyanin accumulation is cell-autonomous and only occurs in those cells infected by the marked virus in which Roseal is expressed. The amount of anthocyanins correlates with viral load (Bedoya et al. 2012). Unlike fluorescent protein markers, pigment accumulation in this system is clearly visible to the naked eye (Majer et al. 2013). We observed that this tool proved quite useless in zucchini (Cucurbita pepo L.) and other cucurbits, ZYMV natural hosts, given a negligible accumulation of colored anthocyanins in infected tissues. However, in experimental host Nicotiana benthamiana Domin, efficient virus-local multiplication in inoculated tissues and an inefficient systemic movement to distal tissues were observed for the recombinant clone. We reasoned that the Roseal-marked ZYMV clone and $N$. benthamiana combination could represent an excellent experimental system to study the virus sequence determinants and the host factors involved in the long-distance movement of this virus. By means of this novel tool, we particularly aimed to analyze the differential contributions of the four $N$. benthamiana DCL genes, core components of the host RNA silencing pathways, in virus systemic movement in $N$. benthamiana. Our results show that, while
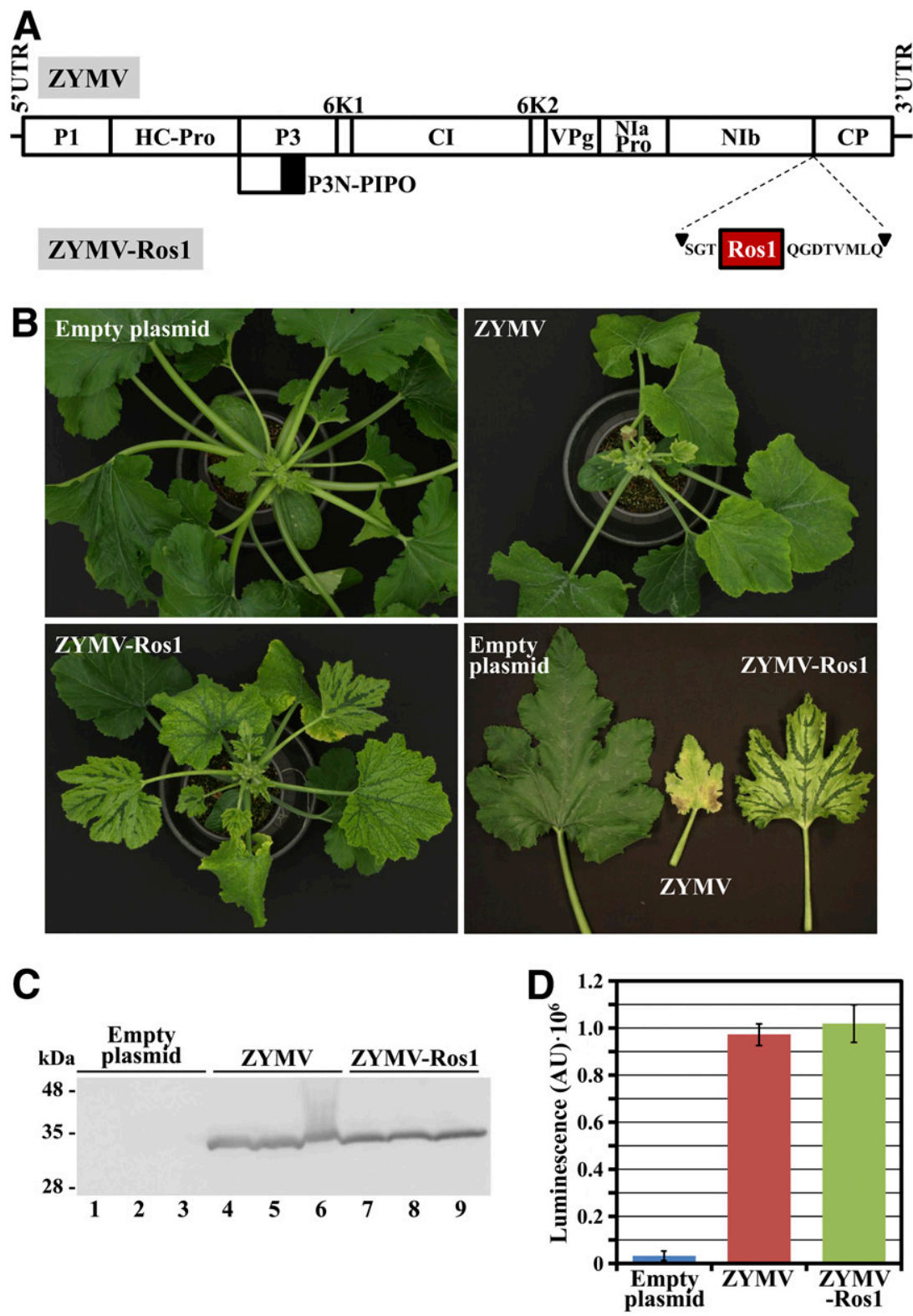

Fig. 1. Symptoms induced by Zucchini yellow mosaic virus (ZYMV) and ZYMV-Ros1 in zucchini plants. A, Schematic representation of the ZYMV genome. Lines represent the $5^{\prime}$ and $3^{\prime}$ untranslated regions (UTR) and boxes represent the different viral cistrons as indicated. In ZYMV-Ros1, a cDNA that codes for Antirrhinum majus Rosea1 was inserted between the NIb (nuclear inclusion b) and CP (coat protein) cistrons. The cDNA included sequences that corresponded to extra amino and carboxy terminal peptides, as indicated, to mediate proteolytic release from the polyprotein. B, Representative zucchini plants agroinoculated with the empty binary plasmid, ZYMV, or ZYMV-Ros1. To better appreciate symptoms, a picture of selected leaves is also shown. All the pictures were taken at 21 days postinoculation (dpi). C, Western blot analysis of ZYMV CP accumulation in upper noninoculated tissues of three independent plants agroinoculated with the empty plasmid (lanes 1 to 3), wild-type ZYMV (lanes 4 to 6), and ZYMV-Ros1 (lanes 7 to 9) at 15 dpi. The position and size (expressed in kiloDaltons) of marker proteins are indicated at the left of the panel. D, Bar graph representing the average ZYMV CP accumulation, quantified as luminescence arbitrary units (AU) by Western blot, in the upper noninoculated tissues of the previously described (C) zucchini plants. Error bars represent the standard error median. 
individual $D C L$ genes barely contributed to the inhibition of virus multiplication in inoculated tissues, DCLA plays a major role in restricting ZYMV systemic movement in $N$. benthamiana.

\section{RESULTS}

\section{A ZYMV infectious clone that includes the visual Roseal marker suboptimally moves long distance in $N$. benthamiana.}

The Rosea1 marker system has been successfully applied to several combinations of viruses and host plants (Bedoya et al. 2012). However, it cannot be considered as a universal system, as it is based on the activity of a heterologous transcription factor on a host endogenous metabolic pathway. We wondered whether this system could be applied to ZYMV to track infection in cucurbitaceous plants. If so, the system would be a most valuable tool to facilitate high-throughput screening for resistance in cucurbit breeding programs. To this end, we cloned a wild ZYMV isolate from a zucchini plant (cv. Scallop), grown in 2013 in Horta de Vera (Valencia, Spain), which presented severe infection symptoms. Two internal cDNA fragments from the viral genome were amplified by reverse transcription-polymerase chain reaction (RT-PCR), whereas the $5^{\prime}$ and $3^{\prime}$ terminal cDNAs were amplified by a rapid amplification of cDNA ends (RACE) strategy. The sequence information from all these cDNAs was used to design a set of primers (Supplementary Table S1) to amplify the whole genome of the Vera isolate of ZYMV in three cDNA fragments flanked by the recognition site of a type-IIS restriction enzyme. Finally, these fragments were assembled into a binary plasmid in which the full-length ZYMV cDNA was flanked by Cauliflower mosaic virus (CaMV) 35S promoter and a 50-nt-long poly(A) stretch followed by the CaMV 35S terminator. This ZYMV cDNA was sequenced and the resulting full-length sequence was deposited in GenBank as the Vera isolate of ZYMV (GenBank accession number KX499498). A standard nt BLAST search displayed the highest identity with a Taiwanese isolate of ZYMV (GenBank accession number AF127929.2) (Lin et al. 2001). An alignment analysis using ClustalW exhibited $94.6 \% \mathrm{nt}$ identity between both sequences with $517 \mathrm{nt}$ differences.

Next, we inserted a cDNA that corresponded to the A. majus Roseal coding region between ZYMV NIb and CP cistrons, to construct the recombinant virus clone ZYMV-Ros1 (Fig. 1A). Roseal cDNA was flanked by sequences that code for amino acids that complement both sides of the native NIb/CP proteolytic site, to mediate the release of Rosea1 from the viral polyprotein (Fig. 1A; Supplementary Fig. S1). The Agrobacterium tumefaciens clones transformed with plasmids to express ZYMV (pGZYMV) or ZYMV-Ros1 (pGZYMV-Ros1) as well as the empty binary plasmid (pG35Z) were used to inoculate zucchini plants (cv. MU-CU-16). All the plants agroinoculated with ZYMV or ZYMV-Ros1 became infected. The pictures taken at 21 days postinoculation (dpi) show the severe symptoms induced by the Vera isolate of ZYMV (Fig. 1B; Supplementary Fig. S2). The plants infected by ZYMVRos1 showed milder symptoms (Fig. 1B). A Western blot analysis showed that accumulation of both viruses in upper noninoculated leaves of zucchini plants was similar (Fig. 1C and D). Unfortunately, the infected tissues of these plants did not show the expected reddish pigmentation that Rosea1 induces in other species. Similar results were obtained with a different cucurbit species, melon (Cucumis melo L.) plants of the Piel de Sapo cv. (Supplementary Fig. S3).

To confirm that recombinant clone ZYMV-Ros1 expressed a functional copy of Rosea1, we agroinoculated $N$. benthamiana plants using the same Agrobacterium tumefaciens cultures. ZYMV replicates with no symptoms in the inoculated leaves of $N$. benthamiana and, depending on the strain, moves systemically and induces latent infection (Desbiez and Lecoq 1997). We previously showed that this species produces intense reddish pigmentation when infected with several viruses that express Rosea1, including other potyviruses like Tobacco etch virus (TEV) (Bedoya et al. 2012). Unlike the tissues agroinoculated with the empty vector or with ZYMV, tissues agroinoculated with ZYMV-Ros1 displayed intense dark red pigmentation at 7 dpi (Fig. 2A; Supplementary Fig. S4). This result indicates that ZYMV-Ros1 expresses a functional Rosea1 transcription factor that efficiently induces accumulation of reddish anthocyanins in $N$. benthamiana. As expected, none of the $N$. benthamiana plants showed infection symptoms. However, a prolonged observation of these plants revealed that, in those agroinoculated with ZYMV-Ros1, some pigmented foci constantly appeared to be scattered on the upper noninoculated leaves (Fig. 2B). This result suggests that the Vera isolate of ZYMV is able to move a long distance in $N$. benthamiana but inefficiently. It is noteworthy that we detected infection foci on the upper noninoculated leaves, due to the vivid pigmentation induced by the Roseal transcription factor. We realized that the combination of the ZYMV-Ros1 clone and $N$. benthamiana could represent a convenient experimental system to analyze elements involved in ZYMV systemic movement.

\section{Analysis of the contribution of the four host $D C L$ genes to ZYMV systemic movement in $N$. benthamiana.}

With this experimental system at hand, we next aimed to study the effect of the four $N$. benthamiana $D C L$ genes, which are core components of host RNA-mediated silencing pathways, on the systemic movement of ZYMV in this species. To this end,
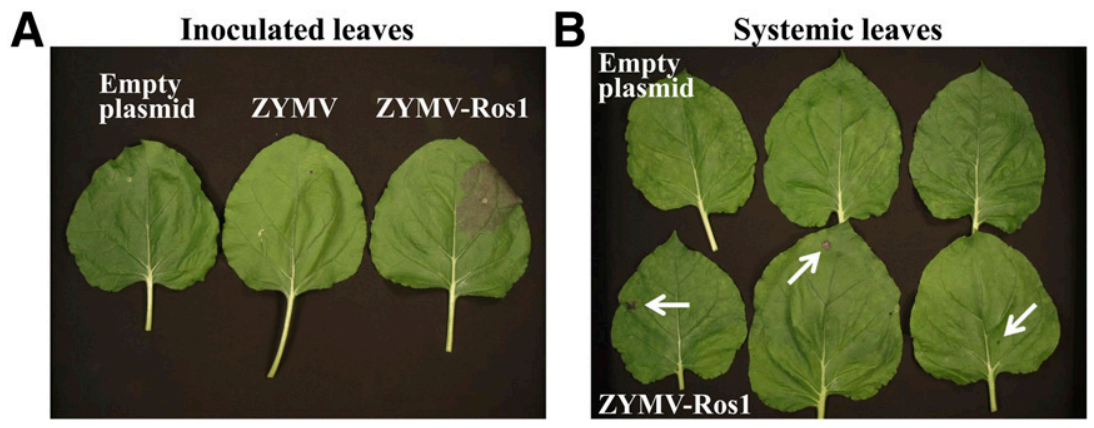

Fig. 2. ZYMV-Ros1 suboptimally moves long distance in Nicotiana benthamiana. A, Leaves agroinoculated with the empty plasmid, Zucchini yellow mosaic virus (ZYMV), or ZYMV-Ros1. Pictures were taken at 7 days postinoculation (dpi). B, Comparison of systemic leaves from three $N$. benthamiana plants agroinoculated with the empty binary plasmid or ZYMV-Ros1. Arrows indicate the pigmented infectious foci induced by ZYMV-Ros1 in systemic leaves. The picture was taken at $21 \mathrm{dpi}$. 
we used a set of $N$. benthamiana transgenic plants in which the different $D C L$ genes were down-regulated by expressing specific hairpin constructs (Dadami et al. 2013; Katsarou et al. 2016). RTquantitative (q)PCR and Northern blot hybridization analyses of transgenic lines DCL1.13i, DCL2.11i, DCL3.10i, and DCL4.9i showed a specific reduction in the DCL1, DCL2, DCL3, and $D C L 4$ mRNAs levels, respectively. Apart from the plant knockdown in the single $D C L$ genes, we also used line DCL2/4.5i, which expresses a hairpin to simultaneously downregulate $D C L 2$ and $D C L 4$, and line DCL3.10 $\times 2 / 4.5 \mathrm{i}$, the heterozygous progeny that results from crossing DCL3.10i as a female and DCL2/4.5i as a male (Dadami et al. 2013; Katsarou et al. 2016).

We first questioned whether these genes had an effect on virus accumulation in inoculated tissue. For this purpose, we agroinoculated two leaves of three $N$. benthamiana plants that corresponded to the wild type and the $D C L$ knockdown lines DCL1.13i, DCL2.11i, DCL3.10i, DCL4.9i, DCL2/4.5i, and DCL3.10 $\times 2 / 4.5 \mathrm{i}$. The agroinoculated tissues were harvested at 6 dpi and proteins were extracted. ZYMV CP was analyzed by electrophoretic separation, followed by Western blot using a specific anti-CP antibody (Fig. 3). Wild-type noninoculated controls were added to the analysis. The quantification of the Western blot signals is summarized in Supplementary Table S2. Figure 3 shows the three Western blots, as well as the graph of ZYMV CP accumulation in the agroinoculated tissue of the different $N$. benthamiana lines. According to the amount of $\mathrm{CP}$, ZYMV-Ros1 accumulation in the inoculated tissues of the DCL1.13i, DCL2.11i, DCL4.9i, and DCL2/4.5i lines was similar to that of the wild-type plants. ZYMV-Ros1 accumulation was lower in the DCL3.10i line (0.6-fold on average) and higher in the DCL3.10 × 2/4.5i line (1.75-fold on average) compared with the wild-type plants (Fig. 3B). However, none of the differences in ZYMV-Ros1 accumulation between wild type and each of the $D C L$ knockdown lines was statistically significant $(P<0.05$ for all pair-wise Student's $t$ test comparisons).

Next, we analyzed the effect of the $D C L$ downregulation on ZYMV systemic movement in $N$. benthamiana. Sets of three plants that corresponded to the wild type and the different knockdown lines were agroinoculated with ZYMV-Ros1 in three leaves. In the $N$. benthamiana lines DCL1.13i, DCL2.11i, and DCL3.10i, we obtained the same outcome previously obtained in the wild-type plants. Very few infection foci were detected in the upper noninoculated tissues. However in $N$. benthamiana line DCL4.9i and in lines DCL2/4.5i and DCL3.10 $\times 2 / 4.5 i$, ZYMV-Ros 1 was able to efficiently move a long distance into the upper noninoculated tissue (Fig. 4A and B). Pictures of the three independent inoculated plants as well as the selected leaves that corresponded to each line are shown in Supplementary Figure S5. We previously showed that anthocyanin accumulation very precisely correlates with viral load in Roseal-marked viruses (Bedoya et al. 2012). Therefore, in order to make a more quantitative estimate of ZYMV-Ros 1 systemic movement in all these plants, we harvested all the aerial tissues above the agroinoculated leaves at $27 \mathrm{dpi}$ and quantified the accumulation of reddish anthocyanins by a spectrophotometric analysis (Fig. 4C; Supplemenatary Table S3). While anthocyanin accumulation in the DCL1.13i, DCL2.11i, and DCL3.10i lines was negligible and indistinguishable from the wild-type plants, the aerial tissues of lines DCL4.9i, DCL2/4.5i, and DCL3.10 $\times 2 / 4.5 \mathrm{i}$ accumulated substantial amounts of these pigments (Fig. 4C). It was noteworthy that the anthocyanin accumulations in the double DCL2/4.5i and in the triple DCL3.10 $\times$ 2/4.5i knockdown lines were 1.8- and 2.9-fold higher, respectively, on average, than in the single knockdown DCL4.9i line. Taken together, these results support a crucial role of DCLA in restricting the systemic movement of ZYMV in $N$. benthamiana that may be functionally complemented by DCL2 and DCL3. To confirm this result, a similar experiment was conducted with wild-type ZYMV under same experimental conditions. Western blot and RTqPCR analyses of virus accumulation at $28 \mathrm{dpi}$ in the whole upper noninoculated tissues confirmed the crucial role of DCL4 in restricting virus systemic movement (Fig. 4D and E).

\section{DISCUSSION}

The goal of this research was to create a tool to facilitate screening ZYMV resistance in the breeding programs of cucurbit plants. Although the initial aim failed, we were able to generate a convenient experimental system to analyze the contribution of ZYMV genetic determinants and host factors to viral systemic movement, which should ultimately help to understand and develop resistance to infection by this virus.

\section{A new Mediterranean isolate of ZYMV}

that mostly resembles a sequence variant from Taiwan.

We constructed an infectious clone from a Spanish isolate of ZYMV that infected a zucchini plant. Our clone mostly resembles sequence variant AF127929.2, which has been reported in Taiwan and was isolated in 1993 from sponge gourd (Luffa cylindrical Roem.) (Lin et al. 2001). The two variants differ in 517 nt positions ( $94.6 \%$ identity), including the insertion of a $U$ at position 9,465 , which corresponds to the $3^{\prime}$ untranslated region. Our finding of a 2013 Mediterranean isolate that mostly resembled a 1993 Taiwanese sequence variant, which belongs to phylogenetic group A-IV, mainly composed of East Asian isolates (Coutts et al. 2011), supports the easy worldwide dispersion of this virus.

\section{The ZYMV-mediated expression of Rosea1 does not produce visible anthocyanin accumulation in cucurbit plants.}

We constructed a recombinant ZYMV clone that expresses A. majus R2R3 MYB transcription factor Rosea1 (ZYMVRos1) (Fig. 1A). This recombinant clone induced the accumulation of reddish anthocyanins in the infected tissues of $N$. benthamiana (Fig. 2) but not in cucurbitaceous species like zucchini (Fig. 1B) or melon. Tomato plants engineered to overexpress the two A. majus transcription factors Rosea1 and Delila under the control of a fruit-specific promoter produced purple tomatoes with high anthocyanin content (Butelli et al. 2008; Su et al. 2016). In plants, anthocyanin biosynthesis is controlled at the transcriptional level by members of three protein families: R2R3 MYB transcription factors, bHLH transcription factors, and WD repeat proteins. They interact to form a ternary complex that activates a series of genes that lead to anthocyanin biosynthesis and accumulation in vacuoles (Zhang et al. 2014). We previously showed that the virus-mediated expression of Rosea1 and Delila in tobacco tissues also induces the accumulation of large amounts of anthocyanins in infected tissues (Bedoya et al. 2010). Next, we reported that the sole virus-mediated expression of Rosea1 suffices to induce pigment accumulation that is readily detectable to the naked eye in infected tissues in several host plant-virus combinations. This finding suggests that this transcription factor is a convenient marker to visually track plant virus infection and movement (Bedoya et al. 2012). In terms of the size, Rosea1 is only slightly larger than the most conventional reporter gene used in plant virology, green fluorescent protein (GFP) (Tilsner and Oparka 2010). Although the impact of Rosea1 in recombinant virus fitness is stronger than that of GFP, the stabilities of both markers in the viral genome are similar (Majer et al. 2013). We succeeded in producing a visible reddish pigmentation of the infected tissues in solanaceous plants ( $N$. benthamiana or N. tabacum L.) and also in the nonsolanaceous Arabidopsis thaliana L., using recombinant potyviruses 
such as TEV or Turnip mosaic virus (TuMV) and also with viruses like Tobacco mosaic virus or Potato virus $X$ that belong to different families (Bedoya et al. 2012). Narcissus mosaic virus (genus Potexvirus) has also been shown to induce visible pigment production in $N$. benthamiana plants when expressing Arabidopsis thaliana R2R3 MYB transcription factor AtMYB75 (PAP1) (Zhang et al. 2013). Lack of pigment accumulation in the tissues of cucurbitaceous plants infected with ZYMV-Ros1 may result from an incompatibility between A. majus Roseal and the endogenous companion transcription factors of the bHLH and WD repeat types. Not much is known about the flavonoid pathway in cucurbits. Other flavonoids, such as flavone derivatives, have been detected in cucumber (Cucumis sativus L.) and melon leaves (Krauze-Baranowska and Cisowski 2001). Flavonol derivatives have also been reported in the reproductive organs of some cucurbits (Imperato 1980). Naringenin chalcone is the main flavonoid that accumulates in the fruit rind of some yellow melon varieties (Feder et al. 2015; Tadmor et al. 2010).

Zucchini plants infected with ZYMV-Ros1 showed milder symptoms than those infected with wild-type ZYMV (Fig. 1B). Some leaves in these plants presented a distinctive beautiful pattern that consisted in dark green perinerval stripes on a lightgreen background (Fig. 1B). The possibility that these distinctive symptoms might still arise from some unknown activity of the Rosea1 transcription factor cannot be ruled out.

\section{ZYMV-Ros1 inefficiently moves long distance in $N$. benthamiana.}

According to the anthocyanin production induced by the Rosea1 marker, we observed that ZYMV-Ros1 efficiently accumulated in the agroinoculated tissues of $N$. benthamiana plants, but very few particles from the progeny were able to establish systemic infection foci (Fig. 2). As an alternative to agroinoculation, we obtained the same result from mechanical inoculation of $N$. benthamiana plants with an extract of ZYMVRos1-infected zucchini. It is worth noting that the visual marker was crucial for this observation, since very few systemic infection foci kept appearing in the first weeks after inoculation. The Rosea1-induced pigmented foci, which were directly observable without using specialized instrumentation such as a UV lamp (Bedoya et al. 2012), easily attracted our attention. It has been previously described that some ZYMV strains induce latent infection in $N$. benthamiana either systemically or in a limited manner to inoculated tissue (Desbiez and Lecoq 1997; Lesemann et al. 1983; Wang et al. 1992).

We reasoned that the combination of our recombinant ZYMVRos 1 virus and the $N$. benthamiana host could represent an excellent experimental system to study the virus genetic determinants and host factors involved in ZYMV systemic movement. $N$. benthamiana, particularly the lineage used in most research laboratories, is susceptible to a large number of plant virus species from very different taxonomic groups. This is most probably because this lineage, which was originally harvested in an extreme habitat of central Australia, is a natural $r d r l$ mutant (Bally et al. 2015; Carbonell 2015). Consequently, this species is frequently adopted as a model plant in many research works into plant viruses. In our system, the amount of viral particles capable of reaching upper noninoculated tissues was easily determined by monitoring the dark red pigmentation of these tissues. The efficiency of viral systemic movement was also quantified in systemic tissues by counting infection foci or by measuring anthocyanin accumulation by a simple colorimetric analysis of methanol extracts. We previously showed that, for Rosea1marked viruses, anthocyanin accumulation correlates with viral load in infected tissues (Bedoya et al. 2012). This experimental system should help to analyze genetic determinants in the virus genome that affect systemic movement. More importantly, this system should allow analyzing the host factors involved in ZYMV systemic movement. In this way, by inoculating knockout or knockdown $N$. benthamiana mutants, host factors involved in favoring or restricting ZYMV systemic movement could be identified and analyzed. Similarly, by inoculating $N$. benthamiana plants in which the candidate factors from cucurbit species are expressed by stable genetic transformation or by transient expression through Agrobacterium tumefaciens or viral vectors,
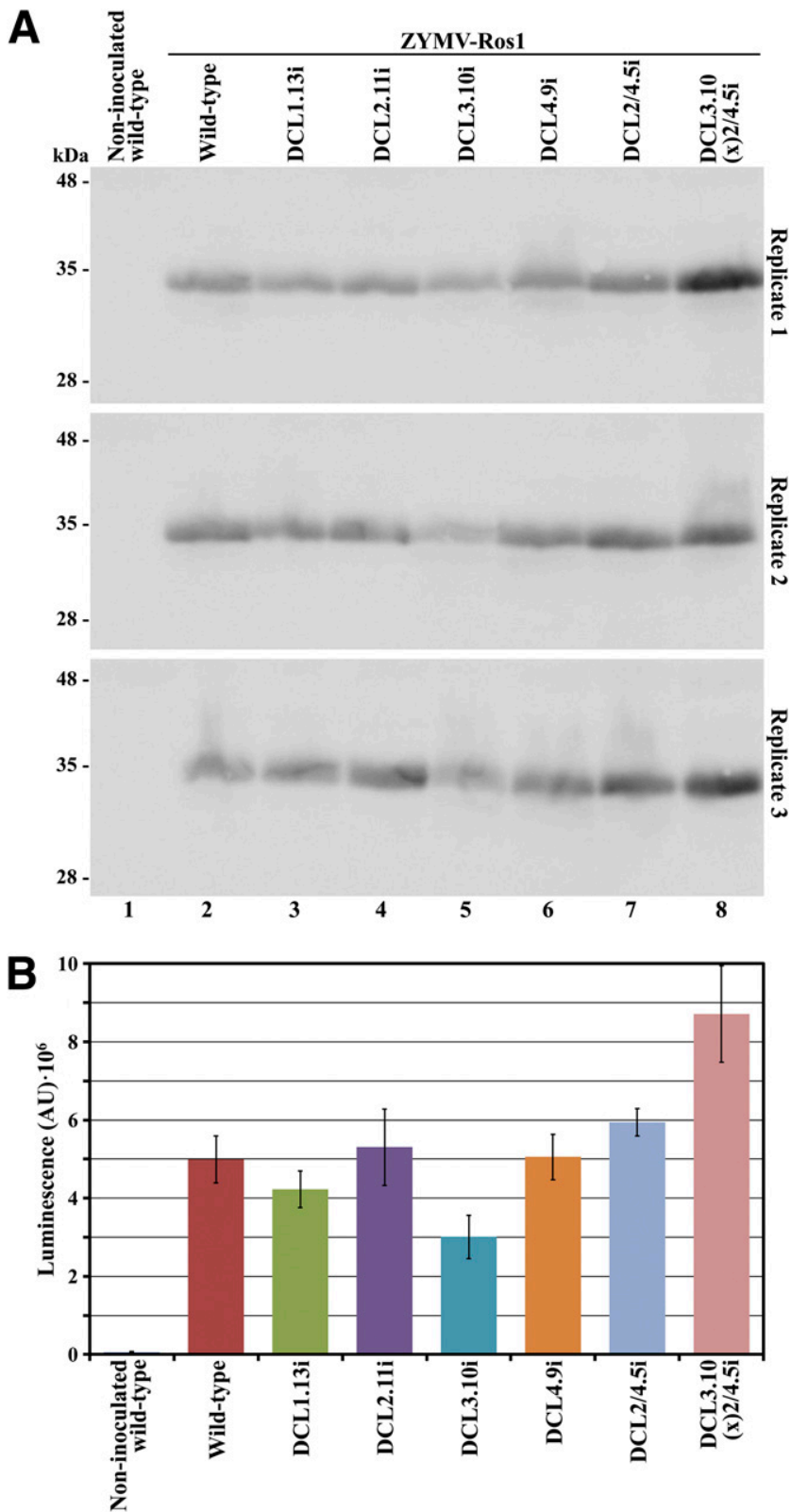

Fig. 3. Accumulation of ZYMV-Ros 1 in the agroinoculated tissues of wildtype Nicotiana benthamiana plants and the lines downregulated in different DCL (Dicer-like) genes. A, Triplicate Western blot analysis of Zucchini yellow mosaic virus (ZYMV) coat protein (CP), using a specific antibody conjugated to alkaline phosphatase and a luminogenic reaction. Proteins were separated by sodium dodecyl sulfate-polyacrylamide gel electrophoresis. Lanes 1 and 2, noninoculated and infected wild-type plants, respectively; lanes 3 to 8 , infected $D C L$ downregulated lines, as indicated. The position and size (in kiloDaltons) of marker is indicated at the left of the panel. B, Bar graph of the average ZYMV CP accumulation, quantified as luminescence arbitrary units (AU), in the agroinoculated tissues of three independent $N$. benthamiana plants that corresponded to the wild-type or $D C L$ downregulated lines, as indicated. Tissue from a noninoculated wild-type plant was also analyzed as a control. Error bars represent the standard error median. 
the host factors that are recruited by the virus to mediate its systemic movement in the natural hosts could be screened. The identification and analysis of all these factors will provide an understanding of the mechanisms that underlie ZYMV systemic movement. These factors may also be used as targets to breed or engineer resistance in cucurbitaceous plants by blocking virus systemic movement.
DCL4 is involved in restricting ZYMV systemic movement in $N$. benthamiana.

Since DCL proteins initiate the antiviral RNA silencing response in plants (Aliyari and Ding 2009; Zhang et al. 2015), we used our new experimental system, based on ZYMV-Ros1 and $N$. benthamiana, to analyze the effects of $D C L$ genes on ZYMV systemic accumulation. N. benthamiana, like Arabidopsis thaliana,

A
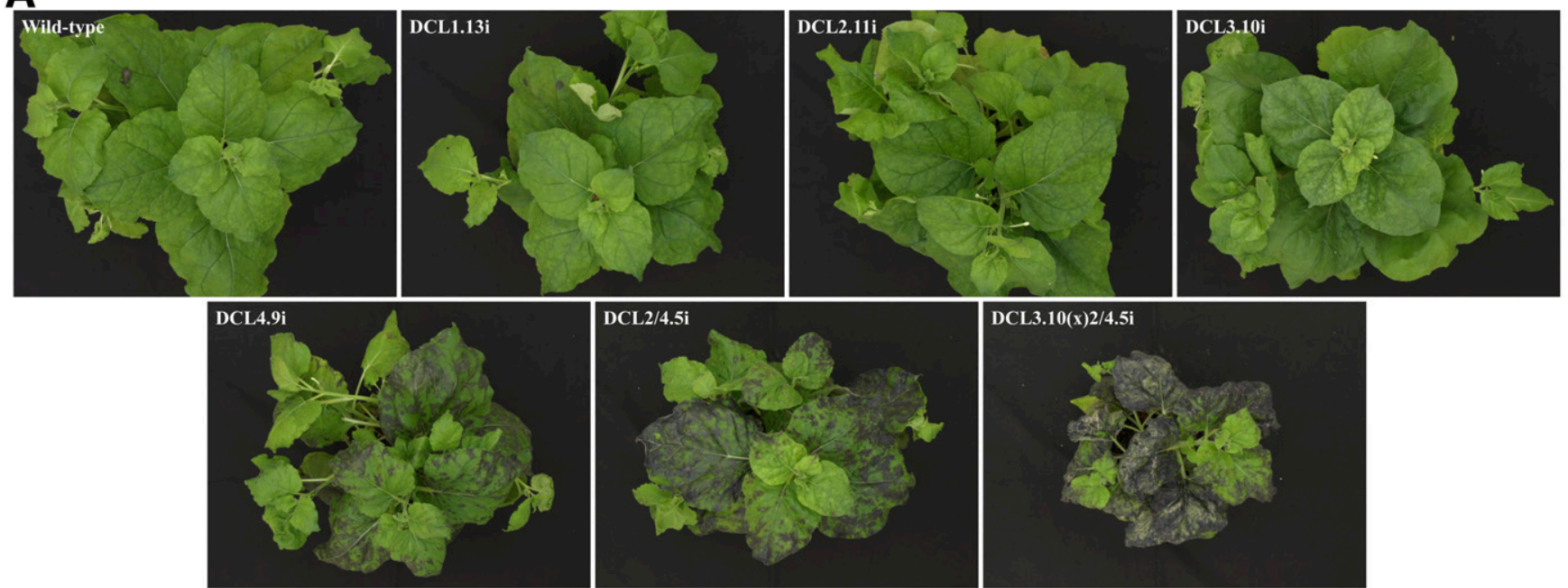

DCL3.10(x)2/4.5i
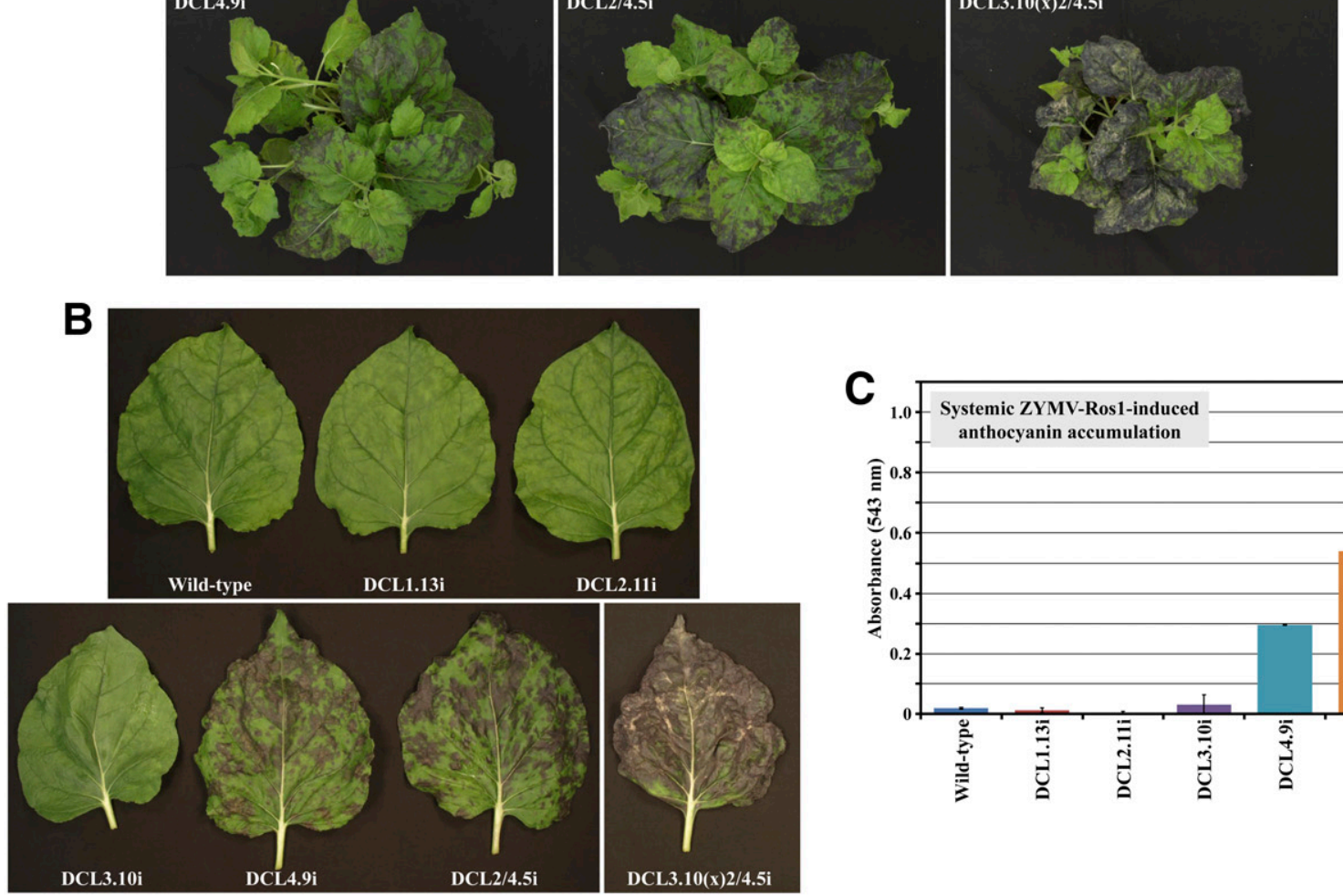

C
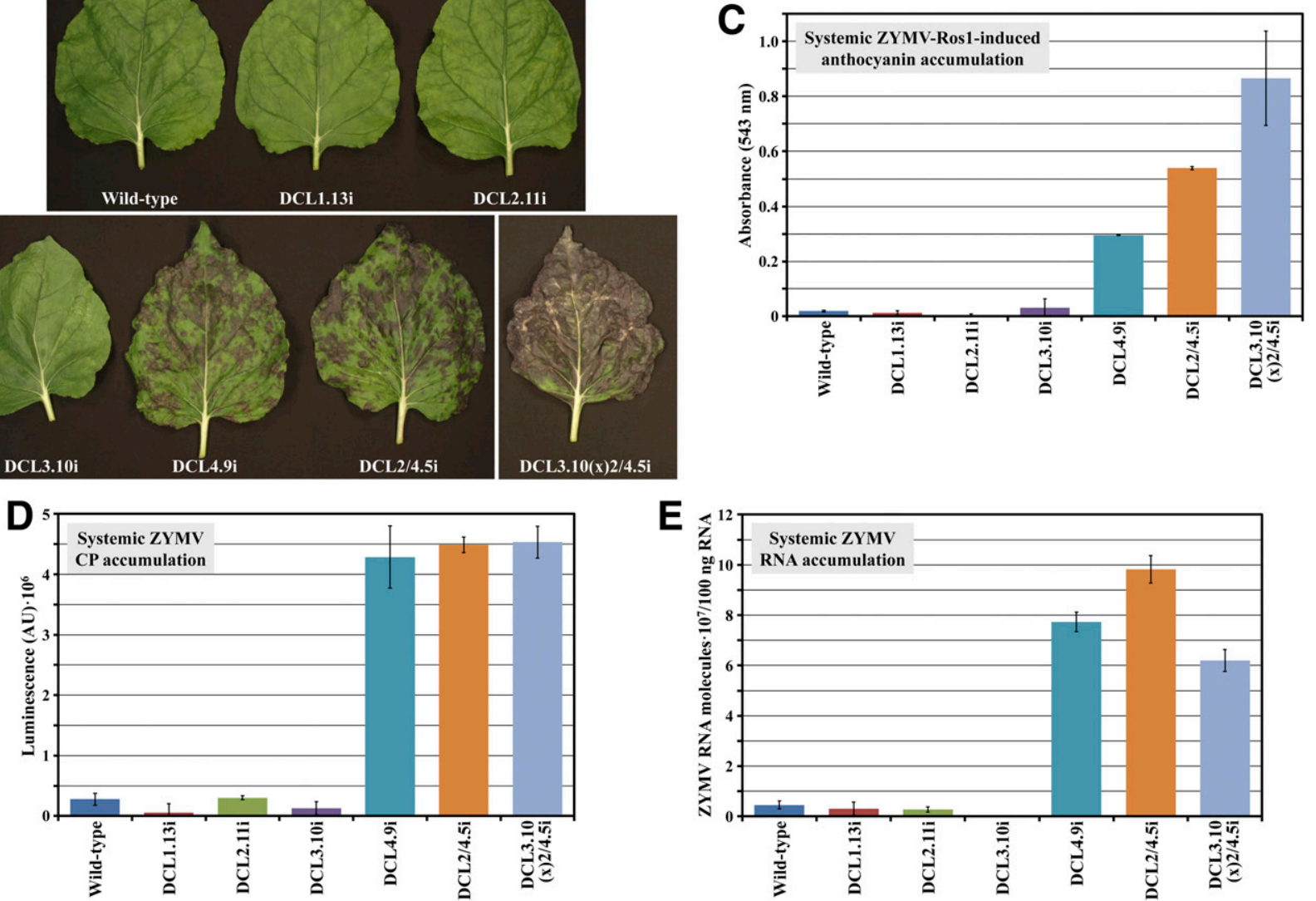

E

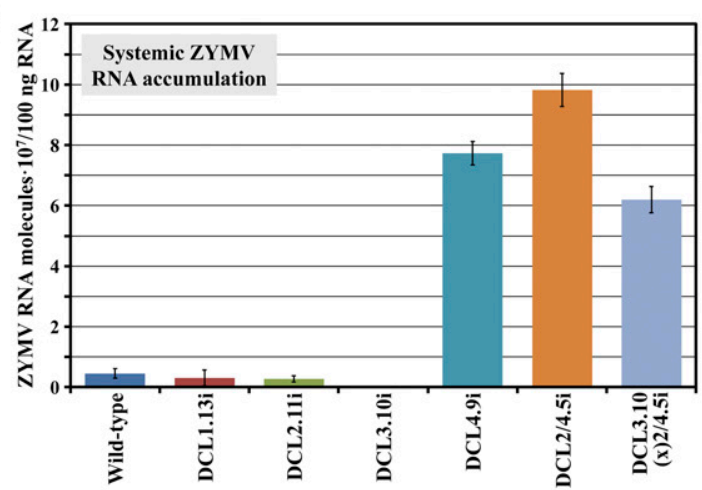

Fig. 4. Zucchini yellow mosaic virus (ZYMV) moves long distance more efficiently in the Nicotiana benthamiana plants in which DCL4 is down-regulated. A, $N$. benthamiana plants that corresponded to the wild-type and DCL knockdown lines, as indicated, and agroinoculated with ZYMV-Ros1. Pictures were taken at 27 days postinoculation (dpi). B, Selected leaves of the plants shown in A. C, Average anthocyanin accumulation, measured as absorbance at 543 nm, in the aerial tissues of three wild-type and DCL knockdown $N$. benthamiana plants, as indicated. The average background absorbance of three wild-type noninoculated controls was subtracted. D, Average ZYMV CP accumulation quantified by Western blot analysis and E, average ZYMV RNA accumulation quantified by reverse transcription quantitative polymerase chain reaction, in the upper noninoculated leaves of three wild-type and $D C L$ knockdown N. benthamiana plants agroinoculated with wild-type ZYMV. Tissues were harvested 28 dpi. Error bars indicated standard error median. 
encodes four DCL type-III RNases (Nakasugi et al. 2013). We took advantage of the availability of a recently generated collection of $N$. benthamiana RNAi transgenic lines, in which the different $D C L$ genes were down-regulated (Dadami et al. 2013; Katsarou et al. 2016). To better understand the role of these genes in ZYMV systemic movement, we first analyzed the effect of their downregulations on ZYMV-Ros1 local multiplication in agroinoculated tissue (Fig. 3). Interestingly, local accumulation of ZYMV-Ros1 was reduced (0.6-fold on average) in DCL3 single knockdown plants compared with that observed in wild-type plants. DCL3, which is primarily involved in antiviral defense against DNA viruses (Akbergenov et al. 2006) but also against RNA viruses as DCL4 surrogate (Deleris et al. 2006; Garcia-Ruiz et al. 2010), could be directly involved in ZYMV-Ros1 genome amplification or cell-to-cell movement or, alternatively, could regulate one or more host factors that favor virus multiplication. DCL3 activity may also have a negative effect on DCL2 and DCL4. In contrast, the single downregulation of DCL1,DCL2, and DCL4 and the double downregulation of DCL2 and DCL4 had no effect on ZYMV-Ros1 local accumulation, which apparently suggests that these three $D C L$ may be dispensable for local antiviral silencing. However, in this context, we should consider that, unlike most studies in which viruses with mutations in silencing suppressors have been used (Ziebell and Carr 2009), in our system, ZYMV-Ros1 expresses a wild-type HC-Pro, which may mask the local antiviral effects of these particular $D C L$, as reported before for TuMV in Arabidopsis thaliana (GarciaRuiz et al. 2010). The local accumulation of ZYMV-Ros1 increased (1.75-fold on average) in the triple DCL2, DCL3, and DCL4 knockdown line compared with wild-type plants. This result suggests that these three antiviral $D C L$ genes possess cooperative antiviral activity against ZYMV-Ros1 in inoculated tissue, as previously observed in other plant-virus systems (Andika et al. 2015; Garcia-Ruiz et al. 2010).

Next, we analyzed the effect of the DCL downregulation on ZYMV-Ros1 systemic accumulation in upper noninfiltrated tissues. It is interesting to note that, while the single downregulation of DCLA did not affect ZYMV-Ros1 local multiplication, it had a dramatic effect by favoring virus accumulation in systemic tissue. This favorable effect was not observed in the single DCL1, DCL2, and DCL3 knockdown plants (Fig. 4). Similar results were obtained using a wild-type ZYMV and analyzing virus systemic accumulation by Western blot (Fig. 4D) or by RT-qPCR (Fig. 4E). Therefore, the observations made for ZYMV-Ros1 are unlikely to be an artifact that resulted from an unexpected activity of the Rosea1 marker. In the case of ZYMV-Ros1, the analysis of the anthocyanin content in upper noninoculated tissues revealed that virus systemic accumulation was enhanced in the double DCL2 and DCL4 and, particularly, in triple $D C L 2, D C L 3$, and DCL4 knockdown plants (Fig. 4). Taken together, these observations support a critical role of DCLA in restricting ZYMV-Ros1 systemic accumulation in $N$. benthamiana, while DCL2 and DCL3 may functionally complement DCLA in this role. Nonetheless, the specific mechanisms that explain how DCL4 hinders ZYMV systemic amplification in $N$. benthamiana still need to be determined. While it is conceivable that DCL4 prevents the entry or passage of viruses into the phloem, DCL4 may also restrict the virus from leaving vascular bundles, as reported for suppressordeficient Turnip crinkle virus in Arabidopsis thaliana (Deleris et al. 2006). In this scenario, as 21-nt siRNA duplexes can move a long distance in plants, the DCL4-dependent 21-nt siRNA duplexes could be the mobile silencing signal generated in inoculated tissue, which spread throughout the plant to prevent ZYMV from accumulating in the upper noninoculated leaves (Mermigka et al. 2016). In any case, viral systemic movement in plants is a rather complex and prolonged process and the specific mechanisms by which antiviral silencing blocks viral systemic spread need to be further clarified.

\section{MATERIALS AND METHODS}

\section{Amplification of ZYMV cDNAs.}

Total RNA was purified by silica gel chromatography (Zymo Research) from a piece of symptomatic leaf from a zucchini plant (cv. Scallop) growing in 2013 in Horta de Vera (Valencia, Spain) that showed typical symptoms of viral infection. From this RNA preparation, cDNAs were initially synthesized using RevertAid RT (Thermo Fisher Scientific) and oligodeoxynucleotide primers $\mathrm{P} 1$ and $\mathrm{P} 4$, designed on the basis of ZYMV GenBank reference sequence variant NC_003224.1. The two cDNAs were amplified with Phusion high-fidelity DNA polymerase (Thermo Fisher Scientific) and primers P2 and P3 and P5 and P6. New cDNAs corresponding to the $5^{\prime}$ and $3^{\prime}$ viral ends were amplified by RACE. To amplify the $3^{\prime}$ end, we took advantage of the native polyadenilate tail of ZYMV genomic RNA. Using primer P7, we synthesized a cDNA that was next amplified by two subsequent PCRs using primers P8 and P9 and P10 and P11. To amplify the $5^{\prime}$ end, we first synthesized a cDNA using primer P12. A polytimidine tail was next added to the $3^{\prime}$ end of this cDNA, using calf thymus terminal transferase (Thermo Scientific). Finally, the 5' end was amplified in two consecutive PCRs using primers P13 and P14 and P15 and P16. All these cDNAs were inserted into EcoRV-digested pBluescript II KS(+) (GeneBank accession number X52327.1) and were sequenced. Experimental sequences served to design new primers (P17 to P29) to amplify the whole ZYMV genome in three fragments $\left(5^{\prime}\right.$, central, and $\left.3^{\prime}\right)$ by RT-PCR. We applied a nested PCR strategy in which $1 \mu \mathrm{l}$ of the first reaction was used as a template for the second reaction. These three ZYMV cDNAs were ligated to EcoRV-digested pBluescript II KS(+), using T4 DNA ligase (Thermo Fisher Scientific), and Escherichia coli DH5 $\alpha$ electroporated with the products of ligations.

\section{Construction of ZYMV infectious clones.}

The cloned cDNAs corresponding to the 5', central, and 3' fragments of the Vera isolate of ZYMV were recovered by digestion with the type-IIS restriction enzyme Eco31I (Thermo Fisher Scientific) from the pBluescript II $\mathrm{KS}(+)$ derivatives (described above) and were assembled (Engler et al. 2009) into the binary vector pG35Z, also digested with Eco31I. pG35Z is a binary vector derived from pCLEAN-G181(GenBank accession number EU186083) (Thole et al. 2007) that we constructed as a previous step to assemble the ZYMV full-length clone. The map and sequence of pG35Z is in Supplementary Fig. S6. The resulting plasmid harboring the full cDNA of the Vera isolate of ZYMV (GenBank accession number KX499498) was named pGZYMV. Using PCR with the Phusion high-fidelity DNA polymerase, digestion with Eco31I, and ligation with T4 DNA ligase, a cDNA corresponding to the coding region of A. majus Roseal transcription factor (GenBank accession number DQ275529.1) was inserted between the NIb and $\mathrm{CP}$ cistrons (positions 8,541 and 8,542 of KX499498). This cDNA was flanked with sequences coding for amino acids to complement the native $\mathrm{NIb} / \mathrm{CP}$ proteolytic site that was split in two. The resulting plasmid was named pGZYMV-Ros1.

\section{Plant agroinoculation.}

Agrobacterium tumefaciens $\mathrm{C} 58 \mathrm{C} 1$ harboring the helper plasmid pCLEAN-S48 (Thole et al. 2007) was electroporated with pG35Z (empty plasmid), pGZYMV, or pGZYMV-Ros1. Liquid cultures of transformed Agrobacterium tumefaciens were grown to optical density at $600 \mathrm{~nm}\left(\mathrm{OD}_{600}\right)$ of approximately 
1.0. Cells were recovered by centrifugation and were resuspended at an $\mathrm{OD}_{600}$ of 0.5 in $10 \mathrm{mM}$ MES-NaOH, pH 5.6, $10 \mathrm{mM} \mathrm{MgCl}_{2}$, and $150 \mu \mathrm{M}$ acetosyringone. Cultures were induced for $3 \mathrm{~h}$ at $28^{\circ} \mathrm{C}$ and were used to agroinoculate zucchini (cv. MU-CU-16), melon (cv. Piel de Sapo), or N. benthamiana plants (Bedoya and Daròs 2010). The inbreeding line MU-CU-16 belongs to the zucchini morphotype of the subspecies pepo of Cucurbita pepo and was provided by the Cucurbits Breeding Group of the Institute for the Conservation and Breeding of Agricultural Biodiversity, Universitat Politècnica de València (Blanca et al. 2011; Esteras et al. 2012). We agroinoculated 3-day-old zucchini, approximately one-month-old melon plants, and 4.5- or 5.5-week-old $N$. benthamiana plants.

\section{Western blot analysis.}

Infiltrated tissues of $N$. benthamiana plants (5.5 weeks old) were harvested 6 dpi (between 0.45 and $0.89 \mathrm{~g}$, depending on the sample) and were ground with a mortar and pestle in the presence of liquid $\mathrm{N}_{2}$. Three volumes of buffer TEW (60 mM Tris-HCl, $\mathrm{pH}$ 6.8, 2\% sodium dodecyl sulfate [SDS], $100 \mathrm{mM}$ dithiothreitol, $10 \% \mathrm{wt} / \mathrm{vol}$ glycerol, and $0.01 \%$ bromophenol blue) were added and the extracts were incubated at $95^{\circ} \mathrm{C}$ for $5 \mathrm{~min}$. Extracts were clarified by centrifugation for $15 \mathrm{~min}$ and $40 \mu \mathrm{l}$ of the supernatants (equivalent to $13 \mathrm{mg}$ of fresh tissue), separated by discontinuous polyacrylamide gel electrophoresis (PAGE), in $12.5 \%$ polyacrylamide gels (5\% polyacrylamide for the stacking gel) containing $0.05 \%$ SDS. Proteins were electroblotted to polyvinylidene fluoride membranes (GE Healthcare), which were blocked for $1 \mathrm{~h}$ in $5 \%$ nonfat milk in buffer WB (10 mM Tris-HCl, pH 7.5, $154 \mathrm{mM} \mathrm{NaCl}$, and $0.1 \% \mathrm{wt} / \mathrm{vol}$ Nonidet P40) and were incubated overnight at $4{ }^{\circ} \mathrm{C}$ with an anti ZYMV CP antibody conjugated to alkaline phosphatase (Agdia) at 1:10,000 dilution in 5\% nonfat milk in WB. Membranes were washed three times with WB and alkaline phosphatase detected with CSPD (Roche Life Science). Luminescence was recorded and quantified with a LAS-3000 image analyzer (Fujifilm). This protocol was also used to analyze the accumulation of ZYMV $\mathrm{CP}$ in upper noninoculated leaves of $N$. benthamiana $D C L$ knockdown plants. In this case, the whole plant aerial tissues were harvested at $28 \mathrm{dpi}$ and were frozen, ground, and mixed. Aliquots of approximately $1 \mathrm{~g}$ of frozen tissue were sampled for the analysis.

\section{Anthocyanins extraction and quantification.}

$N$. benthamiana wild-type and $D C L$ knockdown (Dadami et al. 2013; Katsarou et al. 2016) plants (5 weeks old) were agroinoculated in three leaves with ZYMV-Ros1, as indicated above. The whole aerial parts of the plants were harvested at $27 \mathrm{dpi}$ and were frozen at $-80^{\circ} \mathrm{C}$. Frozen tissues were ground and aliquots of approximately $1 \mathrm{~g}$ were homogenized with 10 volumes of methanol containing $1 \% \mathrm{HCl}$, using a Polytron (Kinematica). Extracts were incubated on ice for $1 \mathrm{~h}$ with occasional vortexing. Extracts were clarified by centrifugation, and an aliquot of the supernatant was further diluted 1:5 in 1\% $\mathrm{HCl}$ in methanol (final ratio of the tissue/extraction solution, 1:50). Anthocyanin concentration was quantified by measuring absorbance at $543 \mathrm{~nm}$ with a spectrophotometer (WPA Biowave II) using a 1-cm path cuvette.

\section{RT-qPCR analysis of ZYMV RNA.}

RNA preparations were purified from $N$. benthamiana tissue samples, using the RNeasy plant mini kit (Qiagen) and were quantified using a NanoDrop ND-1000 spectrophotomer. cDNAs were synthesized in $20-\mu \mathrm{l}$ reactions, including $100 \mathrm{ng}$ of total RNA, 50 U RevertAid RT, and 5 pmol of primer P30. Two microliters of the products of these reactions were subjected to 20- $\mu \mathrm{l}$ qPCR amplification reactions by triplicate, using the Maxima SYBR Green/ROX qPCR master mix (Thermo Scientific) and 6 pmol primers P31 and P32 in a StepOnePlus real-time PCR system (Applied Biosystems). The amount of ZYMV RNA molecules present in $100 \mathrm{ng}$ of RNA preparation was calculated from a calibration line obtained in the same condition with an RNA standard corresponding to the ZYMV 3' genome fragment (from position 8,542 to 9,592 of KX499498), obtained by in vitro run off transcription and quantified by spectrophotometric analysis. StepOne Software v.2.2.2 (Applied Biosystems) was used to analyze the data.

\section{ACKNOWLEDGMENTS}

We thank V. Aragonés for excellent technical assistance. This work was supported by the Spanish Ministerio de Economía y Competitividad (MINECO) through grants BIO2014-54269-R and AGL2013-49919-EXP and by the Greek Ministry for Education and Religious Affairs (Program Aristeia II, 4499, ViroidmiR; ESPA 2007-2013). A. Carbonell was supported by an Individual Fellowship from the European Union's Horizon 2020 research and innovation programme under the Marie SklodowskaCurie grant agreement No. 655841.

\section{LITERATURE CITED}

Akbergenov, R., Si-Ammour, A., Blevins, T., Amin, I., Kutter, C., Vanderschuren, H., Zhang, P., Gruissem, W., Meins, F., Jr., Hohn, T., and Pooggin, M. M. 2006. Molecular characterization of geminivirus-derived small RNAs in different plant species. Nucleic Acids Res. 34:462-471.

Aliyari, R., and Ding, S. W. 2009. RNA-based viral immunity initiated by the Dicer family of host immune receptors. Immunol. Rev. 227:176-188.

Andika, I. B., Maruyama, K., Sun, L., Kondo, H., Tamada, T., and Suzuki, N. 2015. Differential contributions of plant Dicer-like proteins to antiviral defences against potato virus $\mathrm{X}$ in leaves and roots. Plant J. 81: 781-793.

Bally, J., Nakasugi, K., Jia, F., Jung, H., Ho, S. Y., Wong, M., Paul, C. M., Naim, F., Wood, C. C., Crowhurst, R. N., Hellens, R. P., Dale, J. L., and Waterhouse, P. M. 2015. The extremophile Nicotiana benthamiana has traded viral defence for early vigour. Nat Plants 1:15165.

Bedoya, L., Martínez, F., Rubio, L., and Daròs, J. A. 2010. Simultaneous equimolar expression of multiple proteins in plants from a disarmed potyvirus vector. J. Biotechnol. 150:268-275.

Bedoya, L. C., and Daròs, J. A. 2010. Stability of Tobacco etch virus infectious clones in plasmid vectors. Virus Res. 149:234-240.

Bedoya, L. C., Martínez, F., Orzáez, D., and Daròs, J. A. 2012. Visual tracking of plant virus infection and movement using a reporter MYB transcription factor that activates anthocyanin biosynthesis. Plant Physiol. 158:1130-1138.

Blanca, J., Cañizares, J., Roig, C., Ziarsolo, P., Nuez, F., and Picó, B. 2011. Transcriptome characterization and high throughput SSRs and SNPs discovery in Cucurbita pepo (Cucurbitaceae). BMC Genomics 12:104.

Butelli, E., Titta, L., Giorgio, M., Mock, H. P., Matros, A., Peterek, S., Schijlen, E. G., Hall, R. D., Bovy, A. G., Luo, J., and Martin, C. 2008. Enrichment of tomato fruit with health-promoting anthocyanins by expression of select transcription factors. Nat. Biotechnol. 26:13011308.

Carbonell, A. 2015. Molecular ecology: Trading defence for vigour. Nat Plants 1:15174.

Carbonell, A., and Carrington, J. C. 2015. Antiviral roles of plant ARGONAUTES. Curr. Opin. Plant Biol. 27:111-117.

Coutts, B. A., Kehoe, M. A., Webster, C. G., Wylie, S. J., and Jones, R. A. 2011. Zucchini yellow mosaic virus: Biological properties, detection procedures and comparison of coat protein gene sequences. Arch. Virol. 156:2119-2131.

Csorba, T., Kontra, L., and Burgyán, J. 2015. viral silencing suppressors: Tools forged to fine-tune host-pathogen coexistence. Virology 479-480: 85-103.

Dadami, E., Boutla, A., Vrettos, N., Tzortzakaki, S., Karakasilioti, I., and Kalantidis, K. 2013. DICER-LIKE 4 but not DICER-LIKE 2 may have a positive effect on potato spindle tuber viroid accumulation in Nicotiana benthamiana. Mol. Plant 6:232-234.

Deleris, A., Gallego-Bartolome, J., Bao, J., Kasschau, K. D., Carrington, J. C., and Voinnet, O. 2006. Hierarchical action and inhibition of plant Dicer-like proteins in antiviral defense. Science 313:68-71.

Desbiez, C., and Lecoq, H. 1997. Zucchini yellow mosaic virus. Plant Pathol. 46:809-829. 
Engler, C., Gruetzner, R., Kandzia, R., and Marillonnet, S. 2009. Golden gate shuffling: A one-pot DNA shuffling method based on type IIs restriction enzymes. PLoS One 4:e5553.

Esteras, C., Gómez, P., Monforte, A. J., Blanca, J., Vicente-Dólera, N., Roig, C., Nuez, F., and Picó, B. 2012. High-throughput SNP genotyping in Cucurbita pepo for map construction and quantitative trait loci mapping. BMC Genomics 13:80.

Feder, A., Burger, J., Gao, S., Lewinsohn, E., Katzir, N., Schaffer, A. A., Meir, A., Davidovich-Rikanati, R., Portnoy, V., Gal-On, A., Fei, Z., Kashi, Y., and Tadmor, Y. 2015. A kelch domain-containing F-box coding gene negatively regulates flavonoid accumulation in muskmelon. Plant Physiol. 169:1714-1726.

Garcia-Ruiz, H., Takeda, A., Chapman, E. J., Sullivan, C. M., Fahlgren, N., Brempelis, K. J., and Carrington, J. C. 2010. Arabidopsis RNAdependent RNA polymerases and dicer-like proteins in antiviral defense and small interfering RNA biogenesis during Turnip Mosaic Virus infection. Plant Cell 22:481-496.

Hamilton, A. J., and Baulcombe, D. C. 1999. A species of small antisense RNA in posttranscriptional gene silencing in plants. Science 286:950-952.

Imperato, F. 1980. Five plants of the family Cucurbitaceae with flavonoid patterns of pollens different from those of corresponding stigmas. Experientia 36:1136-1137.

Katsarou, K., Mavrothalassiti, E., Dermauw, W., Van Leeuwen, T., and Kalantidis, K. 2016. Combined activity of DCL2 and DCL3 is crucial in the defense against Potato spindle tuber viroid. PLoS Pathog. 12:e1005936.

Krauze-Baranowska, M., and Cisowski, W. 2001. Flavonoids from some species of the genus Cucumis. Biochem. Syst. Ecol. 29:321-324.

Lecoq, H., and Desbiez, C. 2012. Viruses of cucurbit crops in the Mediterranean region: An ever-changing picture. Adv. Virus Res. 84:67-126.

Lesemann, D. E., Makkouk, K. M., Koenig, R., and Natafji Samman, E. 1983. Natural infection of cucumbers by Zucchini yellow mosaic virus in Lebanon. Phytopathol. Z. 108:304-313.

Lin, S. S., Hou, R. F., and Yeh, S. D. 2001. Complete genome sequence and genetic organization of a Taiwan isolate of Zucchini yellow mosaic virus. Bot. Bull. Acad. Sinica 42:243-250.

Lisa, V., Boccardo, G., D’Agostino, G., Dellavalle, G., and D’Aquilio, M. 1981. Characterization of a potyvirus that causes zucchini yellow mosaic. Phytopathology 71:667-672.

Majer, E., Daròs, J. A., and Zwart, M. P. 2013. Stability and fitness impact of the visually discernible Roseal marker in the Tobacco etch virus genome. Viruses 5:2153-2168.

Mermigka, G., Verret, F., and Kalantidis, K. 2016. RNA silencing movement in plants. J. Integr. Plant Biol. 58:328-342.
Nakasugi, K., Crowhurst, R. N., Bally, J., Wood, C. C., Hellens, R. P., and Waterhouse, P. M. 2013. De novo transcriptome sequence assembly and analysis of RNA silencing genes of Nicotiana benthamiana. PLoS One 8:e59534.

Revers, F., and García, J. A. 2015. Molecular biology of potyviruses. Adv. Virus Res. 92:101-199.

Su, X., Xu, J., Rhodes, D., Shen, Y., Song, W., Katz, B., Tomich, J., and Wang, W. 2016. Identification and quantification of anthocyanins in transgenic purple tomato. Food Chem. 202:184-188.

Tadmor, Y., Burger, J., Yaakov, I., Feder, A., Libhaber, S. E., Portnoy, V., Meir, A., Tzuri, G., Sa'ar, U., Rogachev, I., Aharoni, A., Abeliovich, H., Schaffer, A. A., Lewinsohn, E., and Katzir, N. 2010. Genetics of flavonoid, carotenoid, and chlorophyll pigments in melon fruit rinds. J. Agric. Food Chem. 58:10722-10728.

Thole, V., Worland, B., Snape, J. W., and Vain, P. 2007. The pCLEAN dual binary vector system for Agrobacterium-mediated plant transformation. Plant Physiol. 145:1211-1219.

Tilsner, J., and Oparka, K. J. 2010. Tracking the green invaders: Advances in imaging virus infection in plants. Biochem. J. 430:21-37.

Wang, H. L., Gonsalves, D., Provvidenti, R., and Zitter, T. A. 1992. Comparative biological and serological properties of four strains of Zucchini yellow mosaic virus. Plant Dis. 76:530-535.

Wang, X. B., Wu, Q., Ito, T., Cillo, F., Li, W. X., Chen, X., Yu, J. L., and Ding, S. W. 2010. RNAi-mediated viral immunity requires amplification of virus-derived siRNAs in Arabidopsis thaliana. Proc. Natl. Acad. Sci. U.S.A. 107:484-489.

Wu, H. W., Lin, S. S., Chen, K. C., Yeh, S. D., and Chua, N. H. 2010. Discriminating mutations of HC-Pro of zucchini yellow mosaic virus with differential effects on small RNA pathways involved in viral pathogenicity and symptom development. Mol. Plant-Microbe Interact 23:17-28.

Zhang, C., Wu, Z., Li, Y., and Wu, J. 2015. Biogenesis, function, and applications of virus-derived small RNAs in plants. Front. Microbiol. 6: 1237.

Zhang, H., Wang, L., Hunter, D., Voogd, C., Joyce, N., and Davies, K. 2013. A Narcissus mosaic viral vector system for protein expression and flavonoid production. Plant Methods 9:28.

Zhang, Y., Butelli, E., and Martin, C. 2014. Engineering anthocyanin biosynthesis in plants. Curr. Opin. Plant Biol. 19:81-90.

Ziebell, H., and Carr, J. P. 2009. Effects of dicer-like endoribonucleases 2 and 4 on infection of Arabidopsis thaliana by cucumber mosaic virus and a mutant virus lacking the $2 \mathrm{~b}$ counter-defence protein gene. J. Gen. Virol. 90:2288-2292. 